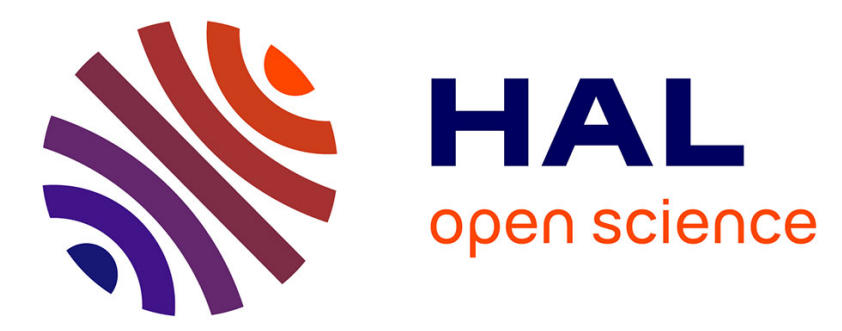

\title{
Optimal Microcell Deployment for Effective Mobile Device Energy Saving in Heterogeneous Networks
}

Kun Wei, Guoqiang Mao, Wuxiong Zhang, Yang Yang, Zihuai Lin, Chung Shue Chen

\section{- To cite this version:}

Kun Wei, Guoqiang Mao, Wuxiong Zhang, Yang Yang, Zihuai Lin, et al.. Optimal Microcell Deployment for Effective Mobile Device Energy Saving in Heterogeneous Networks. IEEE International Conference on Communications (ICC), IEEE, Jun 2014, Sydney, Australia. hal-00947942

\section{HAL Id: hal-00947942 \\ https://hal.inria.fr/hal-00947942}

Submitted on 17 Feb 2014

HAL is a multi-disciplinary open access archive for the deposit and dissemination of scientific research documents, whether they are published or not. The documents may come from teaching and research institutions in France or abroad, or from public or private research centers.
L'archive ouverte pluridisciplinaire HAL, est destinée au dépôt et à la diffusion de documents scientifiques de niveau recherche, publiés ou non, émanant des établissements d'enseignement et de recherche français ou étrangers, des laboratoires publics ou privés. 


\title{
Optimal Microcell Deployment for Effective Mobile Device Energy Saving in Heterogeneous Networks
}

\author{
Kun Wei*†, Guoqiang Mao ${ }^{\ddagger \S}$, Wuxiong Zhang*†, Yang Yang*†, Zihuai Lin ${ }^{* \dagger}$, and Chung Shue Chen $\|$ \\ * Shanghai Institute of Microsystem and Information Technology, Chinese Academy of Sciences, Shanghai, China \\ $\dagger$ Shanghai Research Center for Wireless Communications, Shanghai, China \\ $\ddagger$ The University of Technology, Sydney, Australia \\ $\S$ National ICT Australia, Canberra, Australia \\ I School of Electrical and Information Engineering, University of Sydney, Sydney, Australia \\ I| Smart Wireless Networks, Alcatel-Lucent Bell Labs, Nozay, France
}

\begin{abstract}
Heterogeneous network (HetNet) [1] is considered as an energy efficient system structure to alleviate the problem of rapidly increasing power consumption in the wireless communication system. Significant research on HetNet energy efficiency has been conducted. However, most of them only consider power consumption of Base Stations (BSs) while ignoring influence on energy efficiency of Mobile Devices (MDs) brought by new BSs deployment. In this work, we propose a novel power saving metric for HetNet. Under the coexisting scenario of a single macrocell and a single microcell, we analyze the changes in power consumption at both the BSs side and the MDs side with the deployment of a micro BS. Optimum microcell radii for maximum power saving at the MDs sides and for highest network energy efficiency are obtained through analytical studies. It is found that total power saving for microcell MDs is close to $18 \%$ with a proper deployment of a microcell. Finally, extensive simulations have been provided to establish the accuracy of our theoretical analyses.
\end{abstract}

Index Terms-Heterogeneous network, energy efficiency, microcell planning, power saving

\section{INTRODUCTION}

To address the challenge of rapidly increaing mobile broadband traffic, mobile network service providers have to continuously deploy new BSs. However, the increasing number of BSs will result in higher power consumption, which is becoming a major concern for network service providers due to both economical and environmental considerations. To conquer these challenges, 3GPP suggests the deployment of HetNet as a cost-efficient way to deal with the surging traffic demand and increasing power consumption. HetNet is a network with complex interoperation among macrocell, microcell, picocell, femtocell and WiFi networks, which together provide a mosaic of coverage with handoff capability between network elements [2]. It has been demonstrated both experimentally and theoretically that HetNet provides both a cost-effective and an energy-efficient solution to capacity shortage [3].

This research is partially supported by the NSFC under the grant 61231009, the MOST under grant 2014AA01A707, National Science and Technology Major Project under grant 2011ZX03005-002, Shanghai Municipal Commission of Economy and Informatization R\&D Program-Wireless Communication Technology Test Platform with Shanghai Urban Pattern, Science and Technology Commission of Shanghai Municipality under grant 11JC1412300, and Australian Research Council Discovery projects DP110100538 and DP120102030, and European Celtic project "Operanet2".
In addition to the energy efficiency of the BSs, power consumption reductions of MDs are also increasingly important considerations. More specifically, there are more than six billion mobile phones all over the world, including about one billion smartphones and five billion non-smartphones [4]. It is roughly estimated that the total power consumption of these mobile phones easily exceeds 5 billion kWh/annum. Further, due to the limitation of the battery capacity and the fact that smart phones are much more power consuming, the smart phone usage time is far from satisfactory for most users. A smart phone customer satisfaction studies demonstrated that up to 60 percent of mobile users in China complained short battery usage time as the greatest problem while using $3 \mathrm{G}$ services [5]. The energy efficiency of MDs hence becomes one of the most important considerations for user experience. Therefore an energy efficient network design should not only consider the energy efficient operations of BSs but also take the energy efficiency of MDs into account.

Much prior work has studied the energy efficiency of HetNet, in which different metrics have been defined to measure the energy efficiency of HetNet. The metric area power consumption, which is defined as the average power consumed in a cell divided by the corresponding average size of cellular coverage area, defined by Richter in [9]. Wang and Shen introduced the metric area energy efficiency (with a unit of bit/Joule $/ \mathrm{km}^{2}$ ), which is defined as the number of bits transmitted per joule of energy consumed and per unit area, to measure the energy efficiency of HetNet [8]. This metric has incorporated the impact of the size of cellular coverage area in the analysis of the energy efficiency. In [6], [7], the authors used the value of area energy efficiency divided by the average power consumption of the BSs which is shown in (7) to assess the energy efficiency of HetNet.

With the metrics above, researchers analyzed the energy efficiency of various HetNet BS deployment strategies. In [6], Soh et al. found that the use of small cells generally led to higher energy efficiency but this gain saturated as the density of small cells increases beyond a threshold. In [7], Quek et al. showed that there exists an optimal pico-macro density ratio that maximizes the overall energy efficiency of HetNet. In another work [9], Richter et al. found that in saturated traffic 
load scenarios, the use of micro BSs had a rather moderate effect on the area power consumption of HetNet.

All of the above metrics and associate research focused on studying the energy efficiency of HetNet from service provider's perspective. Comparatively, much less work studied the power consumption of MDs in HetNet and almost all of the work largely ignored the energy efficiency changes of MDs caused by the deployment of new BSs in HetNet. In fact, when new sites are deployed under HetNet, on one hand, the power consumption of MDs will benefit from the increasing number of BSs due to the following two reasons: first, with the increase in the capacity of the HetNet, the MDs will accomplish data transmissions using less amount of time, thus reducing the duration of data transmission which in turn results in reduced power consumption; second, with the increase in the density of BSs, transmission power required by the MDs to reach their respective associate $\mathrm{BSs}$ will reduce, which also leads to reduced power consumption. On the other hand, even if the new sites are low-power devices, the overall power consumption of HetNet BSs may increase [13]. Therefore, from the point of view of power consumption, the increase in the number of BSs in HetNet has two opposite effects on the $\mathrm{BSs}$ and on the MDs respectively. When studying the energy efficiency of HetNet, it is important to jointly consider both of the effects to gain a complete understanding of the energy efficiency of the overall system.

In this paper, we study the energy efficiency of HetNet from a more comprehensive and systematic perspective. Specifically, by considering a HetNet with a single macro BS and a single micro BS, we analyze the power consumption of both at the BSs side and at the MDs side in HetNet system. Based on the analyses, schemes of energy efficient planning of a micro BS are investigated. In summary, contributions of this paper are as follows:

1) A novel power saving metric which considers the power consumption of both BSs and MDs is proposed. The metric helps us to gain a more complete understanding on the energy efficiency of HetNet system.

2) Power consumption of both BSs and MDs under different micro BS deployment strategies is analyzed. Optimum coverage radii of a micro $\mathrm{BS}$ are obtained to maximize the power saving on MDs and to maximize the overall HetNet energy efficiency respectively.

The rest of the paper is organized as follows. In Section II, our system model and the power saving metric are introduced. In Section III, the analyses of power consumption of MDs with and without a microcell $\mathrm{BS}$ and the optimum radius of microcell are provided. Section IV verifies our analyses with extensive simulations. Section V concludes the paper and discusses future research directions.

\section{System Model And Power Saving Metric}

\section{A. System Model}

In this paper, we consider a HetNet model illustrated in Fig. 1 (similar to [10]). Specifically, we consider a HetNet with a single macrocell and a single microcell. The coverage area of the macrocell is modeled by a disk with a radius of $R$,

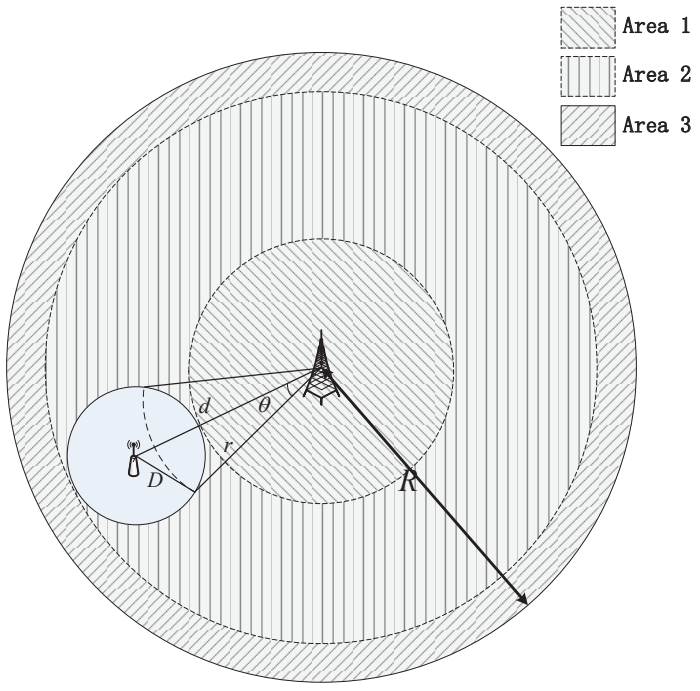

Fig. 1. An Illustration of a HetNet with One Macrocell and One Microcell

in which active MDs are uniformly distributed with density $\lambda$. The coverage area of the microcell is modeled by a disk with a radius of $D$. Denote the Euclidean distance between the macrocell BS and the micro BS by $d$. Downlink scenario is considered in this work. Assume in a certain unit time interval, active MDs need to download a data package with size $S$. Multicarrier deployment strategy, in which the entire spectrum resources are divided into two non-overlapping parts: one used by the macrocell and the other used by the microcell, is adopted in this work [2]. We further assume that all active MDs are allocated with equal bandwidth resources at different carrier frequencies. Therefore there is no interference among MDs. If an MD is located in the coverage area of the micro $\mathrm{BS}$, the MD will be associated with the micro BS; otherwise, the MD will be associated with the macrocell BS.

In addition, a standard power loss propagation model with path loss exponent $\gamma(\gamma \geq 2)$ and path loss constant $L_{0}$ (typically about $(4 \pi / \nu)^{2}$, where $\nu$ is the wavelength) at reference distance $r_{0}=1 \mathrm{~m}$ is adopted [11]. The noise power is assumed to be additive and constant with value $\sigma^{2}$. So the Signal to Noise Ratio (SNR) at an MD which is $x$ meters away from the BS is

$$
S N R=\frac{P_{t x} L_{0} x^{-\gamma}}{\sigma^{2}},
$$

where $P_{t x}$ is the transmission power of the BS. We assume the channel model of macrocell and microcell are the identical as the micro BS in this paper indicates a full functional BS set up in the outdoor area with less coverage size than that of macrocell and larger coverage size than that of picocell for traffic offloading of the network.

The transmission rate of the MD obeys the Shannon's law. Thus if bandwidth for the MD is $B$ and signal to noise ratio is $S N R$, then transmission rate is

$$
\text { Rate }=B \cdot \log _{2}(1+S N R) .
$$


There is a striking contrast in power consumption among different types of MDs [12]. On the basis of the model in [12], the power consumption of MDs is modeled as

$$
P_{p h}=p P_{i}+(1-p) P_{w},
$$

where $P_{i}$ is the power consumption when the MD is in the idle state and $P_{w}$ is the power consumption when the MD is active (i.e., downloading). The $p$ is the probability that the MD is in the idle state.

For base station power consumption, since the power consumption of BS can be approximate as independent of the instantaneous traffic load [13], the consumption models of macrocell and microcell BSs are

$$
\begin{gathered}
P_{m a}=a_{1} P_{t_{m a}}+P_{m_{m a}}, \\
P_{m i}=a_{2} P_{t_{m i}}+P_{m_{m i}},
\end{gathered}
$$

respectively[14], where $a_{1}$ and $a_{2}$ are decided by the efficiency of radio power amplifiers. $P_{t_{m a}}$ and $P_{t_{m i}}$ are transmission powers of the macro BS and micro BS. $P_{m_{m a}}$ and $P_{m_{m i}}$ summarize independent components of transmission power such as signal processing, battery backup, and also parts of the cooling unit.

\section{B. The Power Saving Metric}

Previous work on energy efficiency of HetNet mainly focuses on only one side of the networks (the BSs or the MDs), and the energy efficiency metrics therefore do not give a complete picture of the overall energy efficiency. In fact, power consumption of both BSs and MDs are both essential. When studying the energy efficiency of HetNet, neither side of the two should be neglected. We proposed a novel power saving metric in (6) to capture the energy efficiency of the entire network, including the benefit and cost of deploying new $\mathrm{BSs}$, which is an integration of MDs power consumption and the BSs power consumption. The power saving indicator $\Psi$ is defined as follows: When new BSs are deployed, the difference between the power saved (benefit) at the MDs $\left(P_{\text {save }}\right)$ and a weighted power consumption (cost) increase on the BSs $\left(P_{m i}\right)$ compared with the situation without the new BSs are used for measuring the energy efficiency. The weight $\beta$ is the importance ratio between the power cost and power benefit from mobile network operator's standpoint. As the battery energy for MDs is quite limited and MD usage time is of great significance to user's experience, we think $\beta$ should be less than one to reflect the situation that network operator may accept increased power consumption to improve their user experience.

$$
\Psi=P_{\text {save }}-\beta \cdot P_{m i} .
$$

It can be seen that the larger the $\Psi$ is, the more significant the benefit brought by the deployment of a new micro BS will be. So $\Psi$ provides a useful guidance on the optimum deployment of the new BS is from "power consumption" point of view.
In fact, $\Psi$ is positively correlated with some energy efficiency metrics, such as the widely used metric in [6]

$$
E_{\text {eff }}=\frac{\text { Area Spectral Ef ficiency }}{\text { Average Network Power Consumption }} .
$$

Firstly, high area spectral efficiency will reduce MDs download time, and thus save MDs power, so $P_{\text {save }}$ increases with the increase of Area Spectral Efficiency. Deployment of new BSs with power consumption $P_{m i}$ will increase the Average Network Power Consumption. Compared with the metric $E_{e f f}$, we consider more factors, such as the difference of MD power consumption under different networks and different working status.

\section{ENERGy EFFICIENCY ANALYSES With THE DEPLOYMENT OF A MiCROCELL}

In this section, we firstly analyze the power consumption before and after the deployment of the microcell, then we obtain the optimal value of microcell radius to get the maximum MDs power saving and to reach the best performance.

\section{A. Power Consumption without a Microcell}

Under a single macrocell scenario, the number of macrocell MD is $M_{a}=\lambda \cdot \pi R^{2}$, and thus bandwidth per MD is: $B_{\text {mauser }}=\frac{B_{m a}}{M_{a}}$, where $B_{m a}$ is the total bandwidth of the macrocell.

According to the channel model mentioned in Section II, if minimum SNR required at the edge of the macrocell is $S N R_{R}$, the macrocell BS transmission power is

$$
P_{t m a}=S N R_{R} \cdot \sigma^{2} L_{0}^{-1} R^{\gamma} .
$$

Then the received SNR at a distance of $r$ meters from the $\mathrm{BS}$ is

$$
S N R(r)=\frac{P_{t m a} L_{0} r^{-\gamma}}{\sigma^{2}}=S N R_{R} \cdot\left(\frac{R}{r}\right)^{\gamma} .
$$

The probability density that an MD is located at $r$ meters from the $\mathrm{BS}$ is

$$
p_{m a}(r)=\frac{2 r}{R^{2}} \quad r<R .
$$

Transmission duration of an MD which is $r$ meters away from the macro station is $t_{m a}(r)=\frac{S}{R a_{m a}(r)}$, in which $R a_{m a}(r)$ equals to

$$
R a_{\text {ma }}(r)=B_{\text {mauser }} \cdot \log _{2}\left(1+S N R_{r}(r)\right)=B_{u} \cdot \eta_{r},
$$

where $\eta_{r}$ represents the spectrum efficiency when an MD is located at $r$ meters from the macro BS, namely, $\eta_{r}=\log _{2}(1+$ $S N R(r))$.

Combining (9), (10) and (11), the average transmission duration of all MDs is

$$
\begin{aligned}
T_{m a} & =\int_{0}^{R} \frac{S}{R a_{m a}(r)} \cdot p_{m a}(r) d r \\
& =\int_{0}^{R} \frac{S}{B_{\text {mauser }} \cdot \eta_{r}} \cdot \frac{2 r}{R^{2}} d r .
\end{aligned}
$$


The total power consumption of MDs is

$$
P_{\text {mauser }}=M_{a}\left[P_{w} \cdot T_{m a}+P_{i} \cdot\left(1-T_{m a}\right)\right] .
$$

\section{B. Power Consumption With a Microcell}

For the single macrocell / single microcell HetNet scenario, the active number of macrocell MD is given by $M_{a}^{\prime}=$ $\lambda \pi\left(R^{2}-D^{2}\right)$ and the active number of microcell MD is given by $M_{i}=\lambda \pi D^{2}$. Accordingly, bandwidth of macrocell and microcell MD are $B_{\text {mauser }}^{\prime}=\frac{B_{m a}}{M_{a}^{\prime}}$ and $B_{\text {miuser }}=\frac{B_{m i}}{M_{i}}$. Here, $B_{m i}$ is the total bandwidth of microcell.

Similar as (8), the minimum required SNR at the edge of the microcell is $S N R_{D}$. The microcell BS transmission power is

$$
P_{t m i}=S N R_{D} \cdot \sigma^{2} L_{0}^{-1} D^{\gamma}
$$

Received SNR at distance $r$ from the macro BS is the same as (9). Received SNR at a distance $r^{\prime}$ meters from the micro $\mathrm{BS}$ is

$$
S N R\left(r^{\prime}\right)=\frac{P_{t m i} L_{0} r^{\prime-\gamma}}{\sigma^{2}}=S N R_{D} \cdot\left(\frac{D}{r^{\prime}}\right)^{\gamma} .
$$

As MDs in the coverage of the microcell will associate with the microcell instead after the deployment of microcell, the active macrocell MD distribution will change. When an MD is located in Area 1 and Area 3 shown in Fig. 1, the probability density that the MD is $r$ meters from the $\mathrm{BS}$ is

$$
\begin{gathered}
P\{r \mid M D \text { is in Area } 1\}=\frac{2 r}{(d-D)^{2}}, \\
P\{r \mid M D \text { is in Area } 3\}=\frac{2 r}{R^{2}-(d+D)^{2}},
\end{gathered}
$$

respectively.

In Area 2, for MDs located $r$ meters from the macro station, $\frac{\arccos \left\{\left(d^{2}+r^{2}-D^{2}\right) / 2 d r\right\}}{\pi}$ proportion of the MDs will associate with micro station. So the probability density that a macrocell MD located at the place that is $r$ meters from the macro $\mathrm{BS}$ is

$$
\begin{array}{r}
P\{r \mid M D \text { is inarea } 2\}=\frac{\pi-\arccos \left\{\left(d^{2}+r^{2}-D^{2}\right) / 2 d \cdot r\right\}}{\pi} \\
\times \frac{2 r}{(d+D)^{2}-(d-D)^{2}} \quad d-D<r<d+D .
\end{array}
$$

So from the whole system point of view, in the macrocell, the probability density of active MD as a function of $r$ is given by

$$
p_{m a}^{\prime}(r)=\left\{\begin{array}{l}
\frac{2 r}{\left(R^{2}-D^{2}\right)} \quad r<d-D, r>d+D \\
\frac{\pi-\arccos \left\{\left(d^{2}+r^{2}-D^{2}\right) / 2 d r\right\}}{\pi} \cdot \frac{2 r}{\left(R^{2}-D^{2}\right)} \\
d-D<r<d+D
\end{array} .\right.
$$

The probability density that a microcell MD is located at distance $r^{\prime}$ meters from the micro BS is

$$
p_{m i}\left(r^{\prime}\right)=\frac{2 r^{\prime}}{D^{2}} \quad r<D .
$$

As the transmission rates of both macrocell and microcell MDs ( $R_{\text {mauser }}^{\prime}, R_{\text {miuser }}$ ) obey the Shannon's law (2), the average transmission duration of macrocell and microcell MDs can be derived as

$$
\begin{gathered}
T_{m a}^{\prime}(r)=\int_{0}^{R} \frac{S}{R_{\text {mauser }}^{\prime}(r)} \cdot p_{m a}^{\prime}(r) d r \\
T_{m i}\left(r^{\prime}\right)=\int_{0}^{D} \frac{S}{R_{\text {miuser }}\left(r^{\prime}\right)} \cdot p_{m i}\left(r^{\prime}\right) d r^{\prime} .
\end{gathered}
$$

The sum of macrocell and microcell MDs power consumption can be expressed as

$$
\begin{aligned}
P^{\prime}{ }_{\text {user }}= & M_{a}^{\prime}\left[P_{w} \cdot T_{m a}^{\prime}+P_{i} \cdot\left(1-T_{m a}^{\prime}\right)\right]+ \\
& M_{i}\left[P_{w} \cdot T_{m i}+P_{i} \cdot\left(1-T_{m i}\right)\right]
\end{aligned} .
$$

Then total power saving for MDs is given in Eq. (24) on the next page.

In Equation.(24), $\eta_{r^{\prime}}$ represents the spectrum efficiency when a microcell MD is located at $r^{\prime}$ meters from the micro BS, namely, $\eta_{r}=\log _{2}\left(1+S N R\left(r^{\prime}\right)\right)$.

To make (24) more tractable, we propose an approximation that uses the transmission rate of a certain place, analogous to the well-known "typical node (place)" in stochastic geometry, to represent the average transmission rate of the whole microcell or macrocell MDs. Denote $d_{\text {equal }}$ as the distance from the place to its associated BS and denote $\alpha=\frac{d_{\text {equal }}}{R}$. Through simulations, we found, just as Fig. 2 shows, with different size of coverage, $\alpha$ changed little. Another approximation is that the average transmission rate when the microcell MDs are served by the macrocell before the microcell deployment can be approximated as the transmission rate of the place that is $(d+0.1 D)$ meters from the macro BS. So, denote $\eta_{R \alpha}=$ $\log _{2}\left(1+S N R_{R} \cdot(\alpha)^{-\gamma}\right)$ and $\eta_{D \alpha}=\log _{2}\left(1+S N R_{D} \cdot(\alpha)^{-\gamma}\right)$, (24) can be approximated as

$$
\begin{gathered}
P_{\text {save }} \approx \lambda^{2} \pi^{2} S\left(P_{w}-P_{i}\right)\left[\frac{D^{2} R^{2}}{B_{m a} \eta_{R \alpha}}-\frac{D^{4}}{B_{m i} \eta_{D \alpha}}\right. \\
\left.+\frac{D^{2}\left(R^{2}-D^{2}\right)}{B_{m a} \log _{2}\left(1+S N R_{R} \cdot\left(\frac{d+0.1 \cdot D}{R}\right)-\gamma\right)}\right]
\end{gathered}
$$

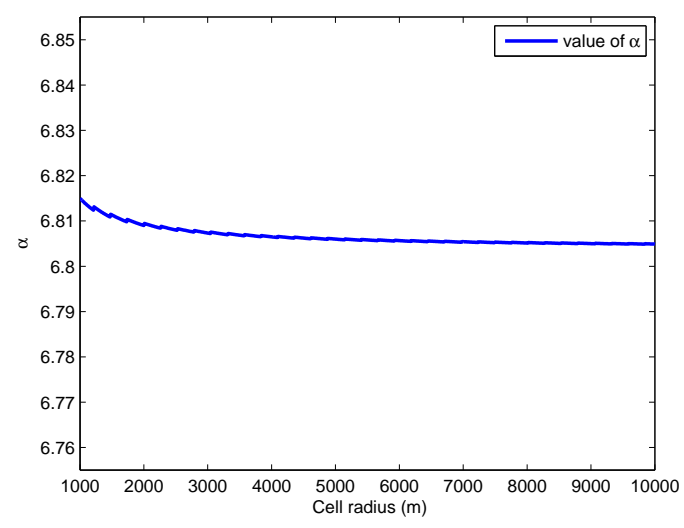

Fig. 2. Equivalent Point $(\alpha)$ over Cell Radius 


$$
\begin{aligned}
& P_{\text {save }}=P_{\text {user }}-P^{\prime}{ }_{\text {user }}=\lambda \pi R^{2}\left(P_{w}-P_{i}\right) \int_{0}^{R} \frac{S}{\frac{B m a}{\lambda \pi R^{2} \cdot \eta_{r}}} \cdot \frac{2 r}{R^{2}} d r-\lambda \pi\left(R^{2}-D^{2}\right)\left(P_{w}-P_{i}\right) \cdot\left[\int_{0}^{d-D} \frac{S}{\frac{B m a}{\lambda \pi\left(R^{2}-D^{2}\right)} \cdot \eta_{r}} \cdot \frac{2 r}{\left(R^{2}-D^{2}\right)} d r\right. \\
& \left.+\int_{d-D}^{d+D} \frac{S}{\frac{B m a}{\lambda \pi\left(R^{2}-D^{2}\right)} \cdot \eta_{r}} \cdot \frac{\pi-a r c \cos \left\{\left(d^{2}+r^{2}-D^{2}\right) / 2 d r\right\}}{2 \pi} \cdot \frac{2 r}{\left(R^{2}-D^{2}\right)} d r+\int_{d+D}^{R} \frac{B}{\frac{B m a}{\lambda \pi\left(R^{2}-D^{2}\right)} \cdot \eta_{r}} \cdot \frac{2 r}{\left(R^{2}-D^{2}\right)} d r-\lambda \pi D^{2}\left(P_{w}-P_{i}\right) \cdot \int_{0}^{D} \frac{S}{\frac{B_{m i}}{\lambda \pi D^{2}} \cdot \eta_{r^{\prime}}} \cdot \frac{2 r^{\prime}}{D^{2}} d r^{\prime}\right] \\
& =\lambda^{2} \pi^{2}\left[D^{2}\left(P_{w}-P_{i}\right) \int_{0}^{R} \frac{S}{B m a \cdot \eta_{r}} \cdot 2 r d r+\left(R^{2}-D^{2}\right)\left(P_{w}-P_{i}\right) \cdot \int_{d-D}^{d+D} \frac{S}{B_{m a} \cdot \eta_{r}} \cdot \frac{a r c \cos \left\{\left(d^{2}+r^{2}-D^{2}\right) / 2 d r\right\}}{\pi} \cdot 2 r d r-D^{2}\left(P_{w}-P_{i}\right) \cdot \int_{0}^{D} \frac{S}{B_{m i} \cdot \eta_{r^{\prime}}} \cdot 2 r^{\prime} d r^{\prime}\right]
\end{aligned}
$$

Denote the term $\lambda^{2} \pi^{2} S\left(P_{w}-P_{i}\right)$ by $K, \frac{1}{\eta_{R \alpha}}$ by $T_{M a}, \frac{1}{\eta_{D \alpha}}$ by $T_{M i}, \frac{1}{\log _{2}\left(1+S N R_{R} \cdot\left(\frac{d+0.1 \cdot D}{R}\right)^{-\gamma}\right)}$ by $T_{d}$, the power saving is

$$
P_{\text {save }}=K\left[\frac{D^{2} R^{2}}{B_{m a}} \cdot T_{m a}-\frac{D^{4}}{B_{m i}} T_{m i}+\frac{D^{2}\left(R^{2}-D^{2}\right)}{B_{m a}} \cdot T_{d}\right] .
$$

From the expression above, we can get some interesting conclusions:

1) Power saving on the MDs side $P_{\text {save }}$ is linearly correlated with the square of the active MD density, down load data size, and the difference of working power and idle power of the MDs.

2) High macrocell capability $\left(B_{m a}\right)$ will impair the benefit of microcell deployment.

3) High microcell capability $\left(B_{m i}\right)$ will save more power on the MDs side.

4) From the parameter $T_{d}$ in the expression, we can see that $P_{\text {save }}$ increases with the increase of distance between the microcell and the macrocell.

\section{The Optimal Microcell Radius to Maximize MDs' Power Saving}

Through (26), we can get the optimal value of $D$ delivering the maximum saving power for the MDs by letting the $\frac{d P_{\text {save }}}{d D}=0$. Ignoring the effect of the $0.1 D$ in $T_{d}$, we can get the optimum value of $D$ as

$$
D=\frac{R}{\sqrt{2}} \sqrt{\frac{T_{m a}+T_{d}}{\frac{B_{m a}}{B_{m i}} T_{m i}+T_{d}}} .
$$

Some interesting results can easily be observed from (27):

1) The optimum $D$ increases linearly with the increase of the macrocell radius $R$.

2) The optimum $D$ is positively correlated with the bandwidth ratio of microcell and macrocell $\left(\frac{B_{m i}}{B_{m a}}\right)$. That is to say, more capable micro BS fits bigger coverage size.

3) The further the microcell is from the macrocell, the bigger the optimum $D$ should be.

\section{The Optimal Microcell Radius for The Highest Power Saving}

According to the definition of the power saving metric in (6), $\Psi$ can be expressed as follows

$$
\begin{aligned}
& \Psi=P_{\text {save }}-\beta \cdot P_{m i} \\
& =K\left[\frac{D^{2} R^{2}}{B_{m a}} \cdot T_{m a}-\frac{D^{4}}{B_{m i}} T_{m i}+\frac{D^{2}\left(R^{2}-D^{2}\right)}{B_{m a}} \cdot T_{d}\right]- \\
& D^{\gamma}\left(\beta \cdot a \cdot S N R_{D} \cdot \sigma^{2} L_{0}^{-1}\right)+\beta \cdot P_{m_{m i}} .
\end{aligned}
$$

TABLE I

SiMULATION PARAMETERS

\begin{tabular}{cc}
\hline Macrocell parameters & \\
\hline Carrier frequency & $2.0 \mathrm{GHz}$ \\
Bandwidth & $20 \mathrm{MHz}$ \\
Path loss model & COST 231 -Walfish-Ikegami Model \\
Radius size & $5 \mathrm{~km}$ \\
Least SNR requirement & $10 \mathrm{~dB}$ \\
Noise & $-160 \mathrm{dBm} / \mathrm{Hz}$ \\
\hline Microcell parameters & \\
\hline Carrier frequency & $2.0 \mathrm{GHz}$ \\
Bandwidth & $2 \mathrm{MHz}$ \\
Path loss model & COST $231-\mathrm{Walfish}-\mathrm{Ikegami}$ Model \\
Least SNR requirement & $10 \mathrm{~dB}$ \\
Noise & $-150 \mathrm{dBm} / \mathrm{Hz}$ \\
\hline MD parameter & \\
\hline Active MD density & $10^{-5} u \mathrm{user} / \mathrm{m}^{2}$ \\
Working power & $1.2 \mathrm{~W}$ \\
Idle power & $0.6 \mathrm{~W}$ \\
Data size (S) & $100 \mathrm{kbit}$ \\
\hline
\end{tabular}

Denote $\frac{a \cdot S N R_{D} \cdot \sigma^{2}}{K L_{0}}$ by $\rho$, then the optimal value of $D$ to balance the energy efficiency of both BSs and MDs will be obtained by setting $\frac{d \Psi}{d D}=0$ :

$$
\begin{aligned}
& \frac{d\left\{K\left[\frac{D^{2} R^{2}}{B_{m a}} \cdot T_{m a}-\frac{D^{4}}{B_{m i}} T_{m i}+\frac{D^{2}\left(R^{2}-D^{2}\right)}{B_{m a}} \cdot T_{d}\right]\right\}}{d D} \\
& -\frac{d\left\{K \beta \cdot \rho D^{\gamma}+\beta \cdot a \cdot P_{m_{m i}}\right\}}{d D}=0 .
\end{aligned}
$$

When $\gamma=2$, the optimal value of $D$ is

$$
D=\sqrt{\frac{R^{2} B_{m i}\left(T_{m a}+T_{d}\right)-B_{m a} B_{m i} \beta \rho}{2\left(B_{m a} T_{m i}+B_{m i} T_{d}\right)}} .
$$

It can be seen that the importance of the power consumption on the $\mathrm{BS}(\beta)$ is negatively correlated with the size of $D$.

\section{NUMERICAL RESULTS}

To verify the accuracy of our analytical results, extensive simulations with the deployment of a microcell with different size and at different location are provided in this section. The simulation parameters are summarized in Table I.

With respect to BS power consumption, some typical parameter values for macrocell and microcell in (4) and (5) are chosen as follows: $a_{\text {macro }}=3.8, P_{m_{m a}}=68.8 \mathrm{~W}$, $a_{\text {micro }}=5.5$ and $P_{m_{m i}}=38 \mathrm{~W}$ respectively [8]. For all the simulations, the coverage of the microcell is assumed to be within that of the macrocell.

\section{A. Numerical Results}

The relationship of power saving at MDs with different microcell size $D$ and different distance between macrocell and microcell $d$ are shown in Fig. 3. In this figure, small circles stand for simulation results and solid lines are analytical results 
according to (26). It can be seen that the two curves are very close to each other. The larger $d$ is, the more power saving can be achieved under the same configurations. This finding coincides with our analytical results in (24) of Section III. For the given configurations, power saving for microcell MDs is close to $18 \%$, compared with the scenario with no microcell deployments. Meanwhile, deployment of microcell also brings about $4 \%$ of power saving for the entire active MDs. If more data are downloaded by MDs, the power saving gain will be more significant.

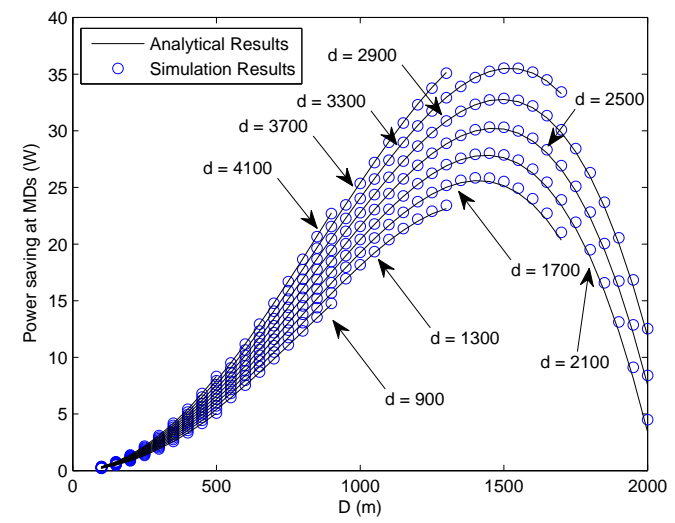

Fig. 3. Power Saving at MDs over Coverage Radius of the Microcell

Fig. 4 shows the power saving indicator of the system measured by the metric in (6). The weight $\beta$ of the power consumption for the mobile network operator is 0.5 . When the $D$ is less than a threshold, about 1400 meters in the figure, with the increasing of $D$, better service of microcell will be of benefit to more and more mobile devices, so the power saving is increasing. However when the microcell radius exceeds the threshold, too many mobile devices are in the microcell, and the inappropriate user assiment will lead to poor performance of the microcell. Thus the power saving will be decreasing with the increasing $D$. If the power saving indicator is larger than 0 , the deployment of a microcell is beneficial from power consumption perspective. Observing from the Fig. 4, with further distance from the macro BS to the micro BS, the power saving improvement by deployment of the micro BS will be more significant.

\section{CONClusion}

In this paper, we analyzed the energy efficiency of HetNets from a more comprehensive and systematic perspective. We proposed an power saving metric which considers the power consumption of both BSs and MDs. Under a single macrocell and a single micro BS scenario, the power saving of the HetNet was analyzed. Approximate expressions which better reveal the relationship of the power consumption at the MDs side as well as the optimum microcell radii with different system parameters of the system were provided. It was shown that the total power saving for microcell MDs is close to $18 \%$ with a proper deployment of a microcell. This work could provide a useful guidance for energy efficient planning of micro BSs.

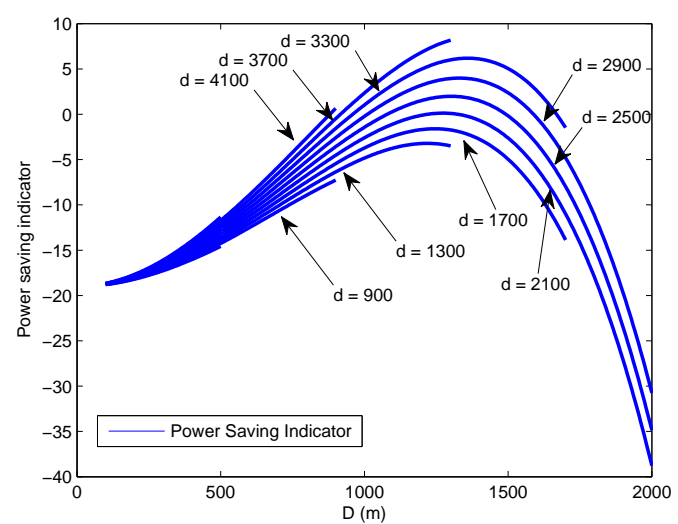

Fig. 4. Power Saving Indicator over Coverage Radius of the Microcell

In the future, more practical MD distribution model and more efficient resource allocation algorithm will be taken into consideration so as to obtain more realistic performance analyses.

\section{REFERENCES}

[1] X. Chu, D. Lopez-Perez, Y. Yang, and F. Gunnarsson, "Heterogeneous Cellular Networks: Theory, Simulation and Deployment," Cambridge University Press, 2013.

[2] A. Damnjanovic, J. Montojo, Y. Wei, T. Ji, T. Luo, M. Vajapeyam, T. Yoo, O. Song, and D. Malladi, "A survey on 3GPP heterogeneous networks," IEEE Wireless Commun., vol. 18, no. 3, pp.10-21, 2011.

[3] R. Q. Hu, Y. Qian, S. Kota, and G. Giambene, "HetNets - a new paradigm for increasing cellular capacity and coverage," IEEE Wireless Commun., vol. 18, no. 3, pp. 8-9, 2011.

[4] ITU 2012 executive summary, "Measuring the information society," http://www.itu.int/dms_pub/itu-d/opb/ind/D-IND-ICTOI-2012-SUMPDF-E.pdf.

[5] D. Feng, C. Jiang, G. Lim , et al., "A survey of energy-efficient wireless communications," IEEE Commun. Surveys \& Tutorials., pp.1-12, no. 99, 2012.

[6] Y. Soh and T. Quek, "Energy efficient heterogeneous cellular networks," IEEE J. Sel. Areas Commun., vol. 31, no. 5, pp. 840-850, 2013.

[7] T. Quek, W. C. Cheung, and M. Kountouris, "Energy efficiency analysis of two-tier heterogeneous networks," Proc. IEEE European Wireless Conf., Vienna, Austria, pp. 1-5, Apr. 2011.

[8] W. Wang and Shen. G, "Energy efficiency of heterogeneous cellular network," Proc. of IEEE Vehic. Technol. Conf., pp. 1-5, Sep. 2010.

[9] F. Richter, A. J. Fehske, and G. P. Fettweis, "Energy efficiency aspects of base station deployment strategies for cellular networks," Proc. of IEEE Vehic. Technol. Conf., pp. 1-5, Sep. 2009.

[10] S. Lee, K. Kim, K. Hong, D. Griffith, Y. H. Kim, and N. Golmie, "A probabilistic call admission control algorithm for WLAN in heterogeneous wireless environment," IEEE Trans. Wireless Commun., vol. 8, no.4, pp. 1672-1676, 2009.

[11] H. S. Jo, Y. J. Sang, P. Xia, and J. G. Andrews, "Heterogeneous cellular networks with flexible cell association: A comprehensive downlink SINR analysis," IEEE Trans. Wireless Commun., vol. 11, pp. 3484-3495, 2012.

[12] L. Zhang and L. Yang., "Accurate online power estimation and automatic battery behavior based power model generation for smartphones," in Proc. ACM International Conference on Hardware/Software Codesign and System Synthesis, pp. 105-114, 2010.

[13] A. Corliano and M. Hufschmid, "Energieverbrauch der mobilen kommunikation," Bundesamt fur Energie, Ittigen, Switzerland, Tech. Rep., in German, Feb. 2008.

[14] ETSI, "Environmental Engineering (EE); Principles for Mobile Network level energy efficiency," ETSI TR 103117 V1.1.1, Nov. 2012. 